\title{
On a problem of Erdős regarding binomial coefficients
}

by

Yossi Moshe (Wien)

1. Introduction and main result. Arithmetical properties of binomial coefficients have been studied by many authors (cf. [1], [3], [4], [5]). Of particular interest is the sequence of middle binomial coefficients $d_{n}=\left(\begin{array}{c}2 n \\ n\end{array}\right)$. Moser [7] proved that no $d_{n}$ is a product of two others. That is, the equation

$$
\left(\begin{array}{c}
2 n \\
n
\end{array}\right)=\left(\begin{array}{c}
2 a \\
a
\end{array}\right)\left(\begin{array}{c}
2 b \\
b
\end{array}\right)
$$

has no solutions with $a, b \geq 1$. Erdős [2] proved that $\left(\begin{array}{c}2 a \\ a\end{array}\right) \nmid\left(\begin{array}{c}2 n \\ n\end{array}\right)$ for each $a \in(n / 2, n)$. This enabled him to show that

$$
\left(\begin{array}{c}
2 n \\
n
\end{array}\right)=\prod_{i=1}^{r}\left(\begin{array}{c}
2 a_{i} \\
a_{i}
\end{array}\right), \quad a_{i} \geq 1,
$$

has no solutions for any $r \geq 2$. In the same paper he raised the following

Question 1 ([2]). Do there exist distinct finite sets $A, B \subseteq \mathbb{N}$ with

$$
\prod_{a \in A}\left(\begin{array}{c}
2 a \\
a
\end{array}\right)=\prod_{b \in B}\left(\begin{array}{c}
2 b \\
b
\end{array}\right) ?
$$

Our main result is

THEOREM 2. For each positive rational number $d$ there exist infinitely many pairs $(A, B)$ of disjoint finite subsets of $\mathbb{N}$ with

$$
\prod_{a \in A}\left(\begin{array}{c}
2 a \\
a
\end{array}\right)=d \prod_{b \in B}\left(\begin{array}{c}
2 b \\
b
\end{array}\right) .
$$

In particular, taking $d=1$ we provide a positive answer for Question 1 .

2000 Mathematics Subject Classification: Primary 11B65, 11D99; Secondary 11A99.

Key words and phrases: binomial coefficients, middle binomial coefficients, diophantine equations.

This research was supported in part by the FWF Project P16004-N05. 
2. Proof of Theorem 2. In this section, all subsets of $\mathbb{N}$ are assumed to be finite (unless explicitly specified otherwise). Given a pair $(A, B)$ of (finite) subsets of $\mathbb{N}$, define

$$
F(A, B)=\frac{\prod_{a \in A}\left(\begin{array}{c}
2 a \\
a
\end{array}\right)}{\prod_{b \in B}\left(\begin{array}{c}
2 b \\
b
\end{array}\right)} .
$$

The main component of our proof is

Proposition 3. Let

$$
\mathcal{G}=\{d \in \mathbb{Q}: \exists A, B \subseteq \mathbb{N}, F(A, B)=d\} .
$$

Then $\mathcal{G}$ is closed under multiplication and division by 2 (i.e., $\left\{2^{l} d_{0}: l \in \mathbb{Z}\right\}$ $\subseteq \mathcal{G}$ for each $\left.d_{0} \in \mathcal{G}\right)$. Moreover, for each $d_{0} \in \mathcal{G}$ there are infinitely many pairs $(A, B)$ of disjoint subsets of $\mathbb{N}$ with $F(A, B)=d_{0}$.

Since $1=F(\emptyset, \emptyset) \in \mathcal{G}$, this already solves Question 1 .

Lemma 4. For every $M \geq 0$ there exist disjoint sets $A, B \subseteq \mathbb{N}$, with $|A|=|B|=3$ and $\min (A \cup B)>M$, such that $F(A, B)=4$.

Proof. Let $n, m, r$ be positive integers and assume that $n, m, r, n-1$, $m-1, r-1$ are distinct. Take

$$
A=\{n, m, r-1\}, \quad B=\{n-1, m-1, r\} .
$$

Observing that $\left(\begin{array}{c}2 t \\ t\end{array}\right)=4\left(1-\frac{1}{2 t}\right)\left(\begin{array}{c}2(t-1) \\ t-1\end{array}\right)$ for each $t>0$, we obtain

$$
F(A, B)=\frac{4(1-1 / 2 n)(1-1 / 2 m)}{1-1 / 2 r} .
$$

Thus, $F(A, B)=4$ if and only if $(1-1 / 2 n)(1-1 / 2 m)=1-1 / 2 r$, that is, $r(2 m+2 n-1)=2 m n$.

Let $k$ be an odd integer and put

$$
n=\frac{k(k-1)^{2}}{4}, \quad m=\frac{k^{2}+1}{2}, \quad r=\frac{(k-1)^{2}}{2} .
$$

Taking a large enough $k$, we see that $r, r-1, n, n-1, m, m-1$ are distinct integers larger than $M$. Note that $2 m+2 n-1=k\left(k^{2}+1\right) / 2$. Thus we get $r(2 m+2 n-1)=2 m n$ and so $F(A, B)=4$.

Proof of Proposition 3. We begin by proving that for each $l \in \mathbb{Z}, M \in \mathbb{N}$ there are infinitely many pairs $\left(\left(A_{n}, B_{n}\right)\right)_{n=1}^{\infty}$ of disjoint subsets of $\mathbb{N}$ with $F\left(A_{n}, B_{n}\right)=2^{l}$ and $\left(A_{n} \cup B_{n}\right) \cap[0, M] \subseteq\{1\}$.

Since $F(B, A)=F(A, B)^{-1}$, we may assume without loss of generality that $l \geq 0$. Write $l=2 t+s$ with $s \in\{0,1\}$. Assume first that $s=0$. Lemma 4 enables us to construct an infinite sequence of pairs $\left(\left(X_{i}, Y_{i}\right)\right)_{i=1}^{\infty}$, with $X_{i}, Y_{i} \subseteq \mathbb{N}, F\left(X_{i}, Y_{i}\right)=4, \min \left(X_{i} \cup Y_{i}\right)>M$, such that $X_{1}, Y_{1}, X_{2}, Y_{2}, \ldots$ 
are pairwise disjoint. If $t>0$ then put

$$
A_{n}=\bigcup_{i=n}^{n+t-1} X_{i}, \quad B_{n}=\bigcup_{i=n}^{n+t-1} Y_{i}, \quad n=1,2, \ldots
$$

Otherwise $t=0$ and put

$$
A_{n}=X_{n} \cup Y_{n+1}, \quad B_{n}=X_{n+1} \cup Y_{n}, \quad n=1,2, \ldots
$$

We conclude that $F\left(A_{n}, B_{n}\right)=4^{t}=2^{l}, A_{n} \cap B_{n}=\emptyset$ and $\min \left(A_{n} \cup B_{n}\right)>M$. The proof for the case $s=1$ is obtained by replacing $A_{n}$ with $A_{n} \cup\{1\}$.

Now let $d_{0} \in \mathcal{G}$, and write $d_{0}=F(A, B)$ with disjoint $A, B \subseteq \mathbb{N}$. Assume first that $1 \notin A \cup B$. Taking $M>\max (A, B)$, we see that $A_{n} \cup B_{n}, A \cup B$ are disjoint, and thus $F\left(A \cup A_{n}, B \cup B_{n}\right)=2^{l} d_{0}$ for each $n$. This completes the proof for this case.

If $1 \in A \cup B$, then the proof is obtained by repeating the same arguments on the triple $\left(A^{\prime}, B^{\prime}, d_{0}^{\prime}\right)$ where $A^{\prime}=A \backslash\{1\}, B^{\prime}=B \backslash\{1\}$ and $d_{0}^{\prime}=F\left(A^{\prime}, B^{\prime}\right)$. (Observe that $d_{0}^{\prime} \in\left\{2 d_{0}, d_{0} / 2\right\}$.)

Lemma 5. For each $c \in\{1,3, \ldots, 15\}, t \in\{1,3\}$ there exist $A, B \subseteq$ $\{1, \ldots, 7\}$ such that $F(A, B)=2^{l} c / t$ for some $l \in \mathbb{Z}$.

Proof. Table 1 provides for each $c \in\{1,3, \ldots, 15\}$ a pair $(A, B)$ with $F(A, B)=2^{l} c$ and a pair $\left(A^{\prime}, B^{\prime}\right)$ with $F\left(A^{\prime}, B^{\prime}\right)=2^{l^{\prime}} c / 3$ for some $l, l^{\prime} \in \mathbb{Z}$.

Table 1. A solution for $F(A, B)=2^{l} c / t$ when $c \in\{1,3, \ldots, 15\}, t \in\{1,3\}$

\begin{tabular}{ccc}
\hline$c$ & $(A, B)$ & $\left(A^{\prime}, B^{\prime}\right)$ \\
\hline 1 & $(\emptyset, \emptyset)$ & $(\emptyset,\{2\})$ \\
3 & $(\{2\}, \emptyset)$ & $(\emptyset, \emptyset)$ \\
5 & $(\{3\}, \emptyset)$ & $(\{3\},\{2\})$ \\
7 & $(\{4\},\{3\})$ & $(\{4\},\{3,2\})$ \\
9 & $(\{3,5\},\{4\})$ & $(\{2\}, \emptyset)$ \\
11 & $(\{2,6\},\{5\})$ & $(\{6\},\{5\})$ \\
13 & $(\{4,7\},\{3,6\})$ & $(\{4,7\},\{2,3,6\})$ \\
15 & $(\{2,3\}, \emptyset)$ & $(\{3\}, \emptyset)$ \\
\hline
\end{tabular}

Given a positive integer $n$, let $[n]_{2}$ denote the binary representation of $n$. Thus, $[n]_{2}=\varepsilon_{t} \ldots \varepsilon_{0}$ is a binary word, with $n=\sum_{k=0}^{t} \varepsilon_{k} 2^{k}$ and $\varepsilon_{t}=1$. Let $\nu(n)$ denote the 2 -adic valuation of $n$ (that is, $2^{\nu(n)}$ is the exact power of 2 dividing $n$ ).

Proof of Theorem 2. Write $d=x / y$ with $x, y \in \mathbb{N}$. A theorem of Kummer [6] implies that for most numbers $k$ (i.e., for a set of density 1 ) we have $y \mid\left(\begin{array}{c}2 k \\ k\end{array}\right)$. Thus, we may take a $k_{0} \geq 8$ such that $\left(\begin{array}{c}2 k_{0} \\ k_{0}\end{array}\right) x / y \in \mathbb{N}$. (In fact, any $k_{0} \geq 8$ with $k_{0} \equiv-1(\bmod y)$ is such.) A simple calculation shows that for any integer $n>0$, the base 2 representations of $n$ and $3 n$ cannot begin with 
the same three letters. In particular, we may take a $K=t\left(\begin{array}{c}2 k_{0} \\ k_{0}\end{array}\right) x / y$ with $t \in\{1,3\}$ so that $\left[k_{0}\right]_{2}$ is not a prefix of $[K]_{2}$. The main part of the proof will be a construction of sets $A_{0}, B_{0}$ such that $\min \left(A_{0} \cup B_{0}\right) \geq 8, k_{0} \notin B_{0}$ and $F\left(A_{0}, B_{0}\right)=2^{l} K / c$ for some $l \in \mathbb{Z}$ and $c \in\{1,3,5, \ldots, 15\}$. Lemma 5 provides sets $A^{\prime}, B^{\prime} \subseteq\{1, \ldots, 7\}$ such that $F\left(A^{\prime}, B^{\prime}\right)=2^{l^{\prime}} c / t$ for some $l^{\prime} \in \mathbb{Z}$. Thus we will get

$$
F\left(A_{0} \cup A^{\prime}, B_{0} \cup B^{\prime} \cup\left\{k_{0}\right\}\right)=2^{l+l^{\prime}} \frac{K}{t\left(\begin{array}{c}
2 k_{0} \\
k_{0}
\end{array}\right)}=2^{l+l^{\prime}} \frac{x}{y} \in \mathcal{G},
$$

and the theorem will then follow by Proposition 3 .

Construct by induction a sequence of odd positive integers $\left(K_{n}\right)_{n=1}^{\infty}$ given by

$$
K_{1}=\frac{K}{2^{\nu(K)}}, \quad K_{n+1}=\frac{K_{n}+1}{2^{\nu\left(K_{n}+1\right)}}, \quad n=1,2, \ldots
$$

If $K_{1} \leq 15$ then the pair $\left(A_{0}, B_{0}\right)=(\emptyset, \emptyset)$ satisfies the required properties (take $\left.c=K_{1}, l=-\nu(K)\right)$. Thus, we may assume that $K_{1}>15$. Note that $K_{n+1}<K_{n}$, unless $K_{n}=1$ (in which case $K_{n+1}=1$ as well). Let $m$ denote the maximal index with $K_{m}>15$. Put

$$
a_{n}=\frac{K_{n}+1}{2}, \quad b_{n}=\frac{K_{n}-1}{2}, \quad n=1, \ldots, m,
$$

and

$$
A_{0}=\left\{a_{1}, \ldots, a_{m}\right\}, \quad B_{0}=\left\{b_{1}, \ldots, b_{m}\right\}, \quad c=K_{m+1} .
$$

Thus $c \leq 15$. Since $K_{m}>15$ we obtain $\min \left(A_{0} \cup B_{0}\right)=b_{m} \geq 8$.

Note that $a_{n}=b_{n}+1$ and thus

$$
\frac{\left(\begin{array}{c}
2 a_{n} \\
a_{n}
\end{array}\right)}{\left(\begin{array}{c}
2 b_{n} \\
b_{n}
\end{array}\right)}=\frac{2\left(2 a_{n}-1\right)}{a_{n}}=2^{2-\nu\left(K_{n}+1\right)} \frac{K_{n}}{K_{n+1}}, \quad n=1, \ldots, m .
$$

Since $a_{1}, b_{1}, a_{2}, b_{2}, \ldots, a_{m}, b_{m}$ are distinct, we conclude that $F\left(A_{0}, B_{0}\right)=$ $2^{l} K_{1} / K_{m+1}=2^{l^{\prime}} K / c$ for some $l, l^{\prime} \in \mathbb{Z}$. It can be easily observed that $\left[b_{n}\right]_{2}$ is a prefix of $[K]_{2}$ for each $n \leq m$. Thus, our assumptions ensure that $k_{0} \notin B_{0}$. This completes the proof.

Acknowledgments. I would like to thank Daniel Berend for introduction to the subject and many useful suggestions.

\section{References}

[1] D. Berend and J. E. Harmse, On some arithmetical properties of middle binomial coefficients, Acta Arith. 84 (1998), 31-41.

[2] P. Erdős, On some divisibility properties of $\left(\begin{array}{c}2 n \\ n\end{array}\right)$, Canad. Math. Bull. 7 (1964), 513518. 
[3] A. Granville, Arithmetic properties of binomial coefficients. I. Binomial coefficients modulo prime powers, in: Organic Mathematics (Burnaby, BC, 1995), CMS Conf. Proc. 20, Amer. Math. Soc., Providence, RI, 1997, 253-276.

[4] A. Granville and O. Ramaré, Explicit bounds on exponential sums and the scarcity of square-free binomial coefficients, Mathematika 43 (1996), 73-107.

[5] N. Kriger, Arithmetical properties of some sequences of binomial coefficients, preprint.

[6] E. Kummer, Über die Ergänzungssätze zu den allgemeinen Reciprocitätsgesetzen, J. Reine Angew. Math. 44 (1852), 93-146.

[7] L. Moser, Notes on number theory. V. Insolvability of $\left(\begin{array}{c}2 n \\ n\end{array}\right)=\left(\begin{array}{c}2 a \\ a\end{array}\right)\left(\begin{array}{c}2 b \\ b\end{array}\right)$, Canad. Math. Bull. 6 (1963), 167-169.

Erwin Schrödinger Institute

Boltzmanngasse 9

A-1090 Vienna, Austria

E-mail: ymoshe@esi.ac.at 